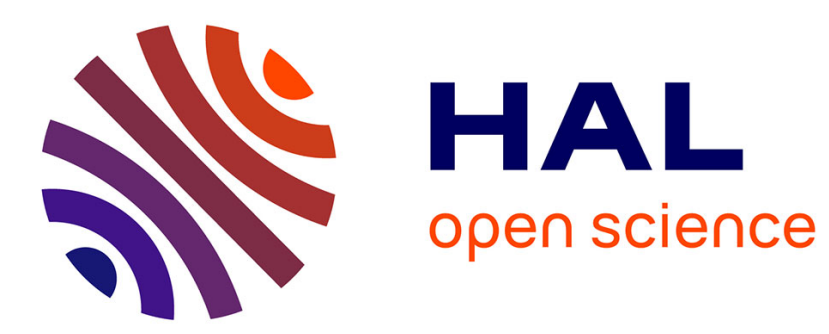

\title{
Variations du coefficient de dilatation linéaire avec la contrainte appliquée dans un alliage métallique amorphe
}

\author{
D. Lesueur, J.Y. Thomas, V. Venditti
}

\section{To cite this version:}

D. Lesueur, J.Y. Thomas, V. Venditti. Variations du coefficient de dilatation linéaire avec la contrainte appliquée dans un alliage métallique amorphe. Revue de Physique Appliquée, 1971, 6 (1), pp.91-94. 10.1051/rphysap:019710060109100 . jpa-00243506

\section{HAL Id: jpa-00243506 https://hal.science/jpa-00243506}

Submitted on 1 Jan 1971

HAL is a multi-disciplinary open access archive for the deposit and dissemination of scientific research documents, whether they are published or not. The documents may come from teaching and research institutions in France or abroad, or from public or private research centers.
L'archive ouverte pluridisciplinaire HAL, est destinée au dépôt et à la diffusion de documents scientifiques de niveau recherche, publiés ou non, émanant des établissements d'enseignement et de recherche français ou étrangers, des laboratoires publics ou privés. 


\title{
VARIATIONS DU COEFFICIENT DE DILATATION LINÉAIRE AVEC LA CONTRAINTE APPLIQUÉE DANS UN ALLIAGE MÉTALLIQUE AMORPHE
}

\author{
D. LESUEUR, J. Y. THOMAS et V. VENDITTI \\ Groupe d'Effets des Radiations, C. E. N. de Fontenay-aux-Roses, 92, France
}

(Reçu le 31 octobre 1970)

\begin{abstract}
Résumé. - Nous décrivons un appareil permettant de mesurer le coefficient de dilatation linéaire d'échantillons de faible épaisseur à des températures au-dessus de la température ordinaire avec ou sans contrainte appliquée. La méthode a été utilisée pour des échantillons d'alliage métallique amorphe Pd-Si. Les valeurs trouvées pour les coefficients de dilatation et le module thermoélastique apparentent cet alliage amorphe plus à un solide qu'à un liquide.
\end{abstract}

Abstract. - An apparatus is described which allows to measure the coefficient of thermal expansion of thin samples at temperatures above room temperature with or without an applied stress. It has been used for amorphous Pd-Si samples. The values of thermal expansion coefficient and of thermoelastic modulus obtained for this amorphous metallic alloy are typical more of a crystalline solid than of aliquid.

1. Introduction. - L'alliage métallique amorphe Pd-Si que nous étudions est obtenu par trempe rapide à partir de l'état liquide. On obtient de cette manière des disques de $1 \mathrm{~cm}$ de diamètre et de $20 \mu$ d'épaisseur environ. Les dilatomètres classiques ne conviennent pas pour des échantillons aussi minces. La méthode décrite ci-dessous permet de mesurer le coefficient de dilatation linéaire d'éprouvettes minces sur lesquelles on applique ou non une contrainte.

2. Description de l'appareillage. - La figure 1 montre que l'appareil comporte un bâti sur lequel est monté un mors fixe. Un mors mobile est relié par l'intermédiaire d'une tige au système de mesure des

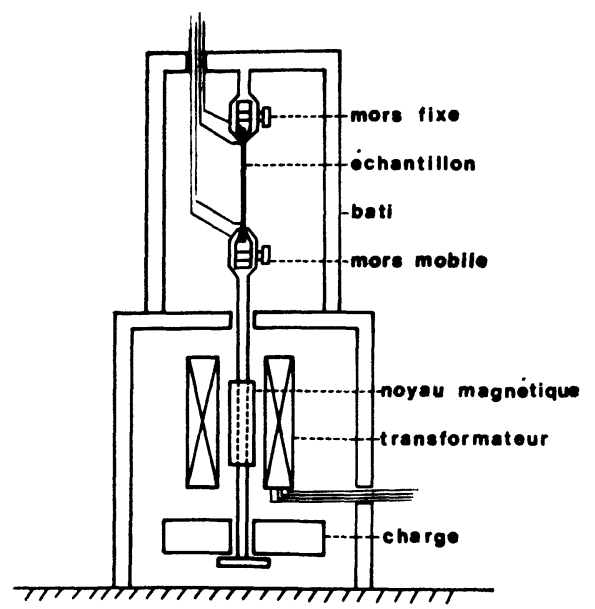

FIG. 1. - Appareil de dilatométrie. allongements. Ces deux parties, bâti et tige, sont réalisées dans une même matière (invar) pour éviter les dilatations différentielles dues aux variations de températures extérieures. Le tout est mis sous vide.

2.1 Montage DE L'ÉChANTILLON. - L'échantillon est un ruban (voir paragraphe 3). Il est pincé dans chaque mors sur lesquels on a collé du mica avec de l'araldite afin d'isoler électriquement et thermiquement l'échantillon du reste de l'appareil. Sur l'échantillon sont soudés par points quatre fils de platine pour les mesures de résistance électrique.

2.2 Mesure des allongements. - Le capteur de déplacement est constitué d'un transformateur linéaire différentiel dans lequel se déplace un noyau de fer doux. Le primaire de la bobine est alimenté par une tension alternative de $1 \mathrm{~V}$ avec une fréquence de $5000 \mathrm{~Hz}$. Les deux enroulements secondaires sont montés en opposition. Le noyau magnétique, solidaire du mors mobile, fait varier en se déplaçant la tension de déséquilibre entre les deux enroulements secondaires C'est cette tension qui constitue le signal proportionnel au déplacement, la constante de proportionnalité étant déterminée par un étalonnage préalable [1]. Ce signal est amplifié, redressé et envoyé sur un enregistreur. On détecte alors aisément $5 \times 10^{-2} \mu$.

2.3 Chauffage. - Le chauffage de l'échantillon n'est pas réalisé par un four car le capteur de déplacement est très sensible aux variations de températures. Le rayonnement dû au four serait suffisant pour per- 
turber les mesures d'allongement. L'échantillon seul est chauffé par effet Joule, le courant de chauffage étant régulé et ajustable de 0 à $10 \mathrm{~A}$.

2.4 MesUre DE LA TEMPÉRATURE. - On trace au préalable une courbe résistance-température de l'échantillon par chauffage dans un four. La température de l'échantillon dans le dilatomètre est alors déterminée par mesure de sa résistance électrique sur un pont de Kelvin. Le chauffage étant réalisé par effet Joule, la température de l'échantillon n'est pas homogène sur toute sa longueur car il y a des fuites thermiques importantes aux deux extrémités. Toutefois on peut mesurer une élévation moyenne de température $\langle\theta\rangle$. $\mathrm{Au}$ début d'une expérience l'échantillon a une température voisine de la température ordinaire, sa longueur est $L_{0}$, sa résistance électrique $R_{0}$, sa résistivité $\rho_{0}$. Désignant par $\theta$ l'élévation de température en un point de l'échantillon, par $s$ la section de l'éprouvette, $\alpha$ le coefficient de dilatation linéaire et $\beta$ le coefficient de température de la résistivité, on a :

Soit

$$
R=\int_{0}^{L_{0}} \rho_{0}(1+\beta \theta) \frac{\mathrm{d} z}{s}(1-\alpha \theta) .
$$

$$
R=R_{0}[1+(\beta-\alpha)<\theta>]
$$

avec

$$
<\theta>=\frac{1}{L_{0}} \int_{0}^{L_{0}} \theta(z) \mathrm{d} z
$$

les éprouvettes ayant une section constante.

De même

$$
L=\int_{0}^{L_{0}}(1+\alpha \theta) \mathrm{d} z=L_{0}(1+<\theta>) .
$$

Il est donc licite de mesurer $L$ en fonction $\langle\theta\rangle$, et de déterminer $\langle\theta\rangle$ en mesurant $R$, ceci suppose seulement que $\alpha$ et $\beta$ soient constants dans le domaine de température considéré.

2.5 PRocÉdure DE MESURE. - On fait passer pendant un temps court (environ 1 minute) un certain

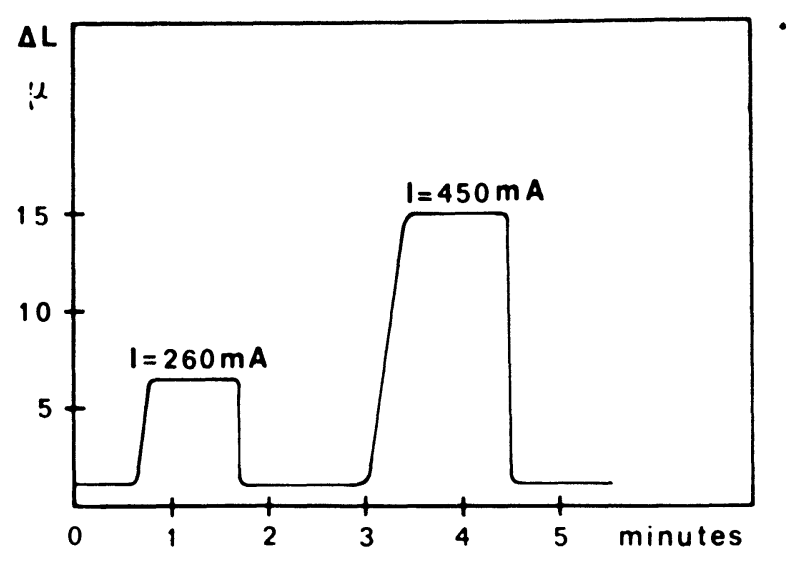

Fig. 2. - Séquence de mesure. courant I dans l'échantillon. Ce dernier grâce à sa faible inertie thermique et à son isolement s'échauffe quasi instantanément. On lit alors sur l'enregistreur la variation de longueur $L-L_{0}$ tandis que l'on mesure la résistance $R$ sur le pont de Kelvin (Fig. 2).

3. Préparation des échantillons. - L'alliage étudié est un alliage Pd-Si comprenant $20 \%$ d'atomes de Si. Il est obtenu sous forme amorphe par trempe rapide depuis l'état liquide [2] et il est stable dans cette phase, qui n'apparaît pas sur le diagramme d'équilibre de cet alliage, jusqu'à environ $350^{\circ} \mathrm{C}$. On peut l'obtenir également sous cette forme en irradiant l'alliage cristallin avec des ions lourds [3, 4]. Nous avons ici étudié l'alliage trempé à l'aide d'un appareil du même type que ceux décrits ailleurs $[5,6,7]$. Dans cet appareil une goutte d'alliage est éjectée du creuset par un jet gazeux et écrasée entre deux flasques métalliques se déplaçant à grande vitesse. On dispose alors d'un disque d'environ $1 \mathrm{~cm}$ de diamètre et de $20 \mu$ d'épaisseur dans lequel est découpée une éprouvette de $1 \mathrm{~mm}$ de large et de $6 \mathrm{~mm}$ de longueur utile.

La courbe résistance-température de cet alliage amorphe est une droite de très faible pente [2]. Sa résistivité est environ $100 \mu \Omega . \mathrm{cm}$. Cette courbe est déterminée pour chaque échantillon par chauffage dans un four ou plus simplement en prenant quelques points fixes entre $77^{\circ} \mathrm{K}$ et $400 \circ \mathrm{K}$. Ceci est indispensable car les conditions de trempe sont peu reproductibles et on obtient d'une trempe à l'autre des coefficients de température de la résistance $\beta^{\prime}=\beta-\alpha$ situés entre $3 \times 10^{-4}$ et $8 \times 10^{-5} \mathrm{oK}^{-1}$.

4. Résultats. - 4.1 CoEfFicients DE DILATATION SANS CONTRAINTE $\alpha_{0}$. - Nous avons mesuré

$$
\frac{\Delta L}{L_{0}}=\frac{L-L_{0}}{L_{0}}
$$

pour différentes valeurs du courant passant dans l'échantillon donc pour différentes valeurs de $\langle\theta\rangle$. Les résultats obtenus (Fig. 3) montrent que $\alpha_{0}$ est bien constant dans le domaine de température considéré. Le tableau I résume les résultats obtenus pour des échantillons ayant des $\beta^{\prime}$ différents.

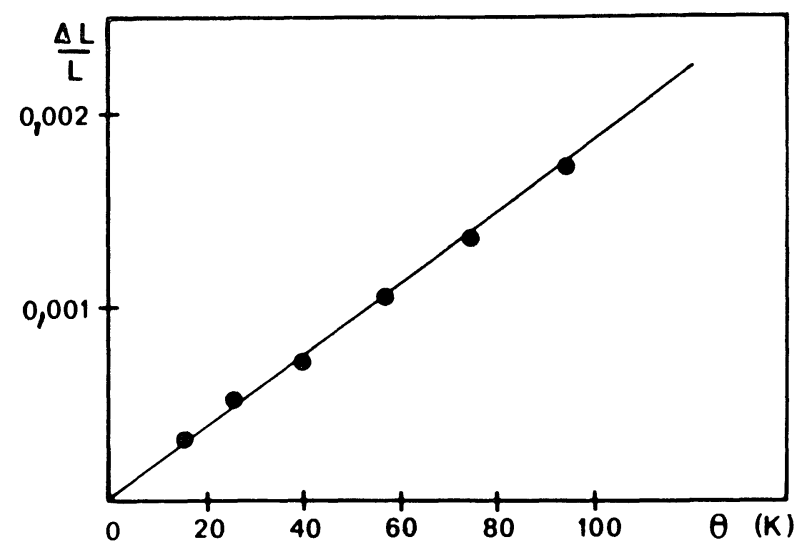

FIG. 3. - Allongement en fonction de la température. 


\section{TABLeaU I}

Coefficient de dilatation linéaire $\alpha_{0}$ et coefficient de température de la résistivité $\beta$ pour 6 échantillons amorphes

\section{Echan-}

\begin{tabular}{|c|c|c|c|}
\hline $\begin{array}{l}\text { llons } \\
\mathrm{n}^{0}\end{array}$ & $\begin{array}{c}\beta^{\prime} \\
\mathrm{o} \mathrm{K}^{-1}\end{array}$ & $\begin{array}{c}\alpha_{0} \\
{ }^{\circ} K^{-1}\end{array}$ & $\underset{\mathrm{o} \mathbf{K}^{-1}}{\beta}$ \\
\hline - & - & - & - \\
\hline 1 & $7,35 \times 10^{-5}$ & $1,08 \times 10^{-5}$ & $8,43 \times 10^{-5}$ \\
\hline 2 & $1,01 \times 10^{-4}$ & $1,95 \times 10^{-5}$ & $1,20 \times 10^{-4}$ \\
\hline 3 & $1,61 \times 10^{-4}$ & $1,73 \times 10^{-5}$ & $1,79 \times 10^{-4}$ \\
\hline 4 & $1,79 \times 10^{-4}$ & $1,64 \times 10^{-5}$ & $1,95 \times 10^{-4}$ \\
\hline 5 & $2,08 \times 10^{-4}$ & $2,05 \times 10^{-5}$ & $2,28 \times 10^{-4}$ \\
\hline 6 & $2,55 \times 10^{-4}$ & $2,75 \times 10^{-5}$ & $2,82 \times 10^{-4}$ \\
\hline
\end{tabular}

Etant donné la précision du pont de Kelvin l'erreur sur $\beta^{\prime}$ est de l'ordre de $0,5 \%$. La très faible valeur absolue de $\beta^{\prime}$ implique une erreur sur $\langle\theta\rangle$ de l'ordre de $2{ }^{\circ} \mathrm{K}$ lorsque l'augmentation de température moyenne est de $100^{\circ} \mathrm{K}\left({ }^{*}\right)$. La source d'erreur la plus importante porte sur la connaissance de la longueur initiale de l'éprouvette entre les points de pincement sur les mors. On peut estimer que l'erreur sur $\alpha_{0}$ est de l'ordre de $10 \%$. Compte tenu de ces erreurs on constate que $\alpha_{0}$ varie dans le même sens que $\beta$ de façon significative.

\subsection{COEFFICIENT DE DILATATION SOUS CONTRAINTE} $\alpha_{\sigma}$ - Nous avons refait les mêmes mesures avec l'échantillon no 2 en lui appliquant des charges différentes en deçà de la limite élastique. On mesure $\alpha_{\sigma}$ et on constate que $\alpha_{\sigma}$ varie linéairement avec la charge (Fig. 4).

Lors de l'application des charges à l'échantillon la mesure de la déformation $\varepsilon$ donne le module de Young $Y$. Il est de l'ordre de $5300 \mathrm{~kg} / \mathrm{mm}^{2}$.

De la différence $\alpha_{\sigma}-\alpha_{0}$ on peut tirer les variations de $Y$ avec la température [8]

$$
\alpha_{\sigma}-\alpha_{0}=-\frac{\sigma}{Y}\left(\frac{1}{Y} \frac{\mathrm{d} Y}{\mathrm{~d} T}\right)
$$

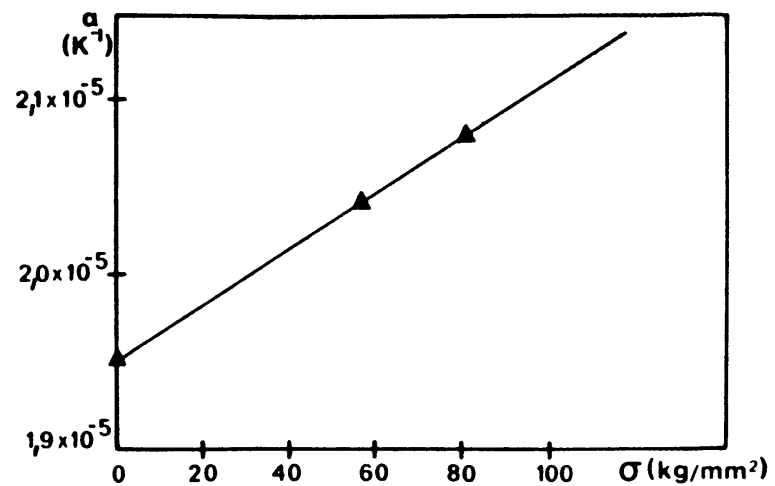

Fig. 4. - Coefficient de dilatation linéaire avec la charge.

On trouve ici

$$
-\frac{1}{Y} \frac{\mathrm{d} Y}{\mathrm{~d} T} \approx 9 \times 10^{-5} K^{-1}
$$

Enfin de la même expérience on tire une valeur $\kappa$ du coefficient d'élastorésistance

$$
\kappa=\frac{1}{\varepsilon} \frac{\Delta R}{R_{0}} .
$$

$\Delta R$ est la variation isotherme de résistance électrique consécutive à la déformation $\varepsilon$. Cette variation est due d'une part à la variation du facteur de forme de l'éprouvette soit $(1+2 v), v$ étant le coefficient de Poisson, d'autre part à la variation de résistivité induite par la déformation.

Pour les échantillons amorphes étudiés on a obtenu $\kappa=1,7 \pm 0,1$. Si le coefficient de Poisson est voisin de 0,3 on voit que $\kappa$ est très peu différent de $1+2 v$ et que par conséquent la résistivité de l'alliage amorphe ne varie pratiquement pas avec la déformation.

4.3 COMPARAISON AVEC DES MÉTAUX ORDINAIRES. Le tableau II donne les valeurs des grandeurs $\beta, \alpha$, $Y,-\frac{1}{Y} \frac{\mathrm{d} Y}{\mathrm{~d} T}$ et $\kappa$ mesurées sur l'alliage amorphe

\begin{tabular}{|c|c|c|c|c|c|c|c|c|}
\hline & $\mathrm{Cu}$ & $\mathrm{Ag}$ & $\mathrm{Al}$ & $\mathrm{Fe}$ & $\mathbf{P d}$ & $\mathrm{Pt}$ & W & $\begin{array}{l}\mathrm{Pd}_{80} \mathrm{Si}_{20} \\
\text { amorphe }\end{array}$ \\
\hline $\begin{array}{c}\beta \times 10^{4} \\
\left({ }^{\circ} \mathrm{K}^{-1}\right)\end{array}$ & 43 & $\overline{35}$ & $\overline{44}$ & $\overline{50}$ & $\overline{38}$ & $\overline{39}$ & $\overline{45}$ & 1 \\
\hline $\begin{array}{c}\alpha \times 10^{5} \\
\left({ }^{\circ} \mathrm{K}^{-1}\right) \\
Y\end{array}$ & 1,7 & 1,9 & 2,5 & 1,1 & 1,1 & 0,9 & 0,43 & 1,5 \\
\hline$\left(\mathrm{kg} \cdot \mathrm{mm}^{-2}\right)$ & 12000 & 7750 & 7000 & 20600 & 11800 & 16600 & 35500 & 5300 \\
\hline $\begin{array}{c}-\frac{1}{Y} \frac{\mathrm{d} Y}{\mathrm{~d} T} \times 10^{5} \\
\left({ }^{\circ} \mathrm{K}^{-1}\right)\end{array}$ & 30 & & 43 & 26 & 10 & 13 & & 9 \\
\hline$\kappa$ & 2,3 & & & 1,2 & & 2,0 & 2,6 & 1,7 \\
\hline
\end{tabular}

TABleaU II

Comparaison entre l'alliage Pd-Si amorphe et quelques métaux

(*) Rappelons que pour un métal comme $\mathrm{Cu}, \beta^{\prime}$ est égal à $4,33 \times 10^{-3}{ }^{\circ} \mathrm{K}^{-1}$. 
$\mathbf{P d}_{80} \mathrm{Si}_{20}$ comparées aux valeurs des mêmes grandeurs dans quelques métaux.

Ce tableau montre que pour $\alpha$ et $-\frac{1}{Y} \frac{\mathrm{d} Y}{\mathrm{~d} T}$ l'alliage amorphe se comporte comme les métaux ordinaires. En revanche ce matériau est plus élastique ( $Y$ est assez faible). Par ailleurs la faible valeur de $\beta$ montre que la résistivité due au désordre l'emporte largement sur la contribution de l'agitation thermique comme dans un métal liquide (la valeur de $\rho \simeq 100 \Omega$.cm est comparable à celle des métaux liquides).
Enfin la valeur de $\kappa$ est un peu plus petite que celle des métaux $[9,10]$, le fer excepté.

Conclusion. - On pourrait s'attendre à trouver pour des échantillons d'alliages métalliques amorphes des coefficients de dilatation linéaires élevés comme c'est le cas des métaux liquides. Il n'en est rien, et on constate au contraire que, si on excepte la résistivité électrique, les propriétés physiques étudiées ici apparentent cet alliage amorphe plus à un solide qu'à un liquide.

\section{Bibliographie}

[1] Weinberg (C.), Conte (R. R.), Dural (J.), Note C. E. A., N-1315 (1970).

[2] Duwez (P.), Willens (R. H.), Crewdson (R. C.), J. Appl. Phys. 1965, 36, 2267.

[3] Lesueur (D.), C. R. Acad. Sci. Paris, 1968, 266,, B 1038.

[4] LesueUr (D.), Fizika (à paraître).
[5] Duwez (P.), Willens (R. H.), Trans. A.I. M.E., $1963,227,362$

[6] Pietrokowsky (P.), Rev. Sci. Instrum., 1963, 34, 445.

[7] DiXmier (J.), GuinIER (A.), Mem. Scient. Rev. Metall., $1967,64,52$.

[8] Quéré (Y.), Ponsoye (J.), Rad. Effects, 1969, 1, 141.

[9] PONSOYE (J.), (à paraître) 1971.

[10] Von Stebut (J.), Communication personnelle. 